\title{
Data reconstruction of homogeneous turbulence using Lagrangian Particle Tracking with Shake-The-Box and machine learning
}

\author{
Dong Kim, Kyung Chun Kim
}

School of Mechanical Engineering, Pusan National University, 46241 Busan, South Korea

This paper proposes a data reconstruction of homogeneous turbulent flow combined machine learning (ML) approach using experimental Lagrangian Particle Tracking (LPT) data with Shake-The-Box (STB). The LPT with STB was adopted to measure a von Kármán flow with a homogeneous turbulent region in the center [1]. The STB results have been stored and a temporal filter using 3rd order B-splines has been applied with optimal weighting coefficients to be used as input for FlowFit data assimilation method [2]. FlowFit data was used as ground truth to train ML algorithm. The low-resolved data of the velocity and acceleration field was reconstructed using an Adaptive Neuro-Fuzzy Inference System (ANFIS) with the downsampled LPT data as an input to predict homogeneous turbulent flow [3]. The training process can be mathematically regarded as an optimization problem to determine the weighting factor.

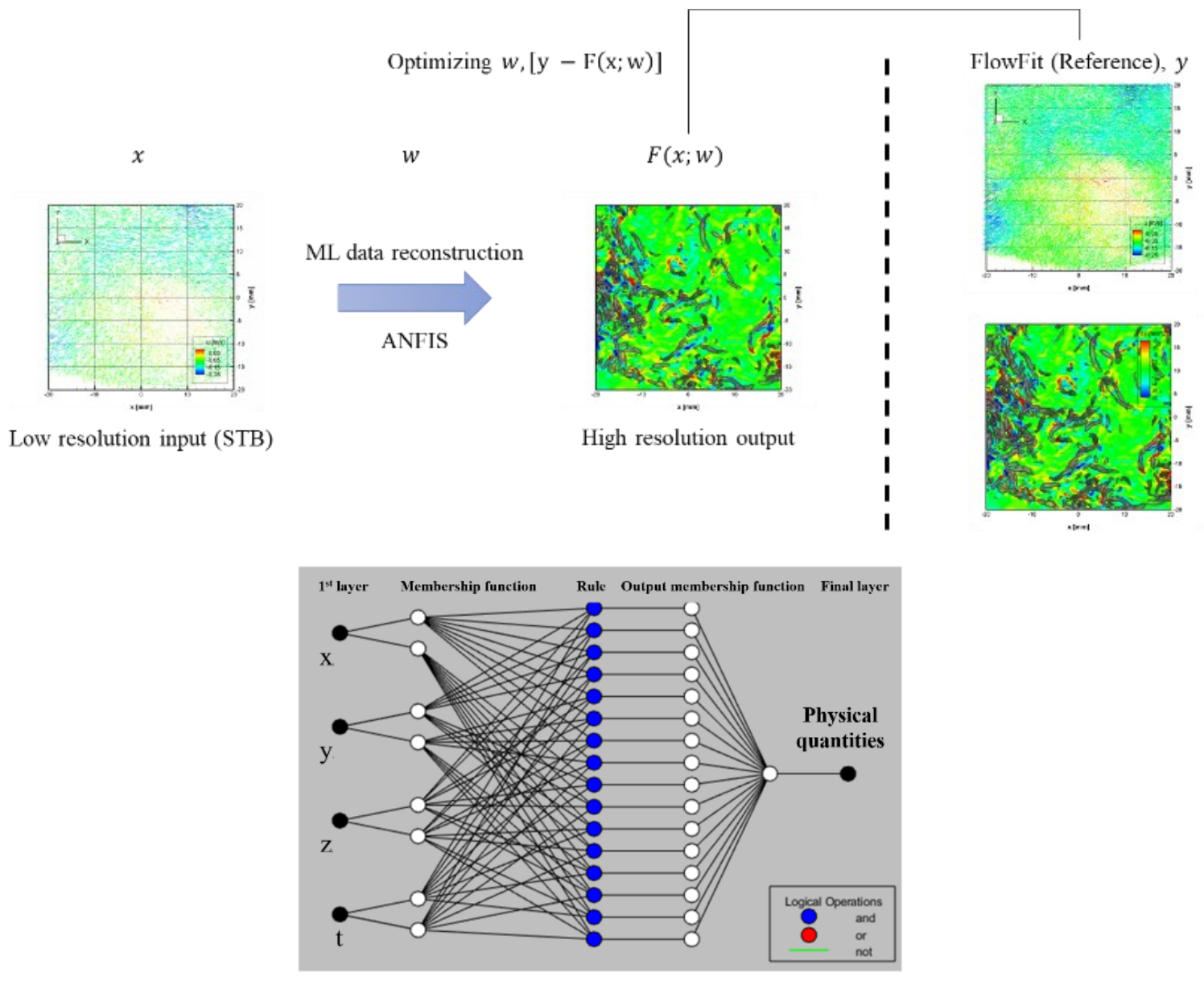

Fig. 1 Data reconstruction methods and ANFIS learning structure. 
Given the input data set $x$ (low resolved STB data) and the desired output data set $y$ (FlowFit results), we aim to find the optimal weight $w$ in a machine-learned model $F$ that acts as a nonlinear regression function such that $F(x ; w) \approx y$. In the present case, $x$ and $F(x ; w)$ represent the lowresolution and reconstructed high-resolution data, respectively. The weight $w$ is optimized between the desired high-resolution output $y$ and the ML model output $F(x ; w)$ is minimized. The ANFISbased data assimilation was first trained with FlowFit data assimilation result as ground truth. Four ANFIS training inputs on the $\mathrm{x}, \mathrm{y}, \mathrm{z}$ coordinates, and time $\mathrm{t}$ of FlowFit results were assigned to $1^{\text {st }}$ layer of ANFIS algorithm. The training targets on velocity, acceleration components were assigned to final layer of ANFIS algorithm to get weighting factor. The computations were performed on a computer with an Intel® Core ${ }^{\mathrm{TM}}$ i5-8250U CPU @ $1.60 \mathrm{GHz} 1.80 \mathrm{GHz}$ and $8.0 \mathrm{~GB}$ of RAM. With 300 epochs and 4 input membership functions, the ANFIS training satisfied the convergence criterion of RMSE $<$ 0.01 . The training time takes 2 hours.

Figure 2 shows the spatial data reconstruction of ANFIS model with different ratio of raw particle density, $\rho_{p}$. The particle density was downsampled by random reduction from raw STB data $(\sim 100,000$ particles to 75,50 ). Compared to FlowFit, ANFIS model can be well reconstructed above $50 \%$ particle density. It revealed much more small-scale vortical structures. Machine learning based data assimilation provide a better understanding of the small turbulence structures and allow for more in-depth analysis by recovering data due to the resolution limit of the experiment. To provide a full turbulence spectrum, full time-series of STB results will later be trained and in-depth analyzed for ANFIS training.
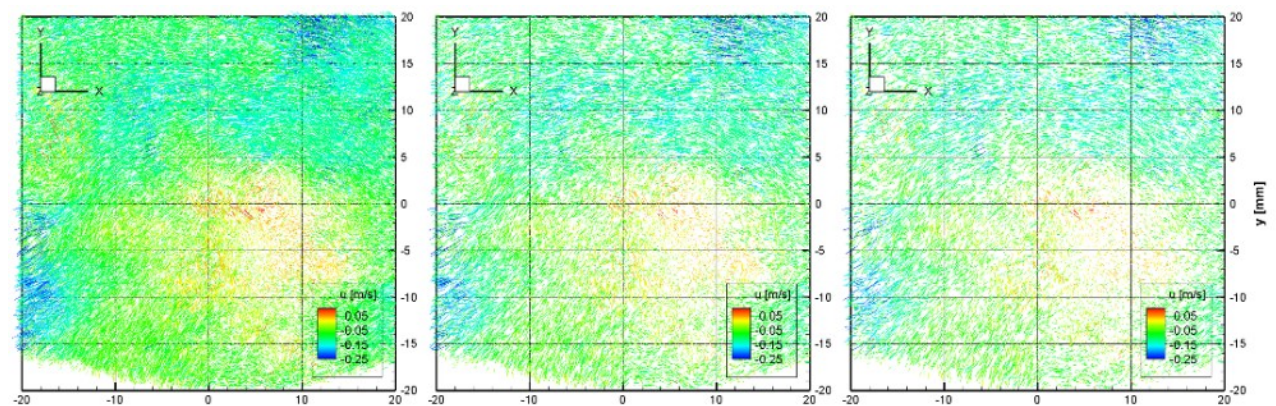

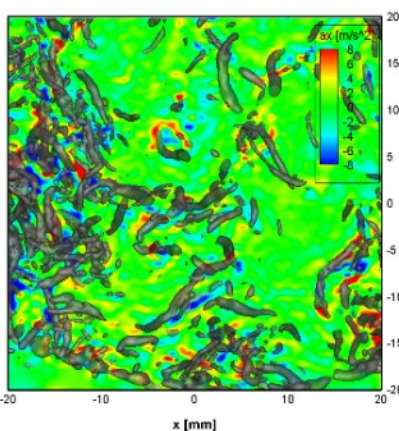

(a) STB, FlowFit

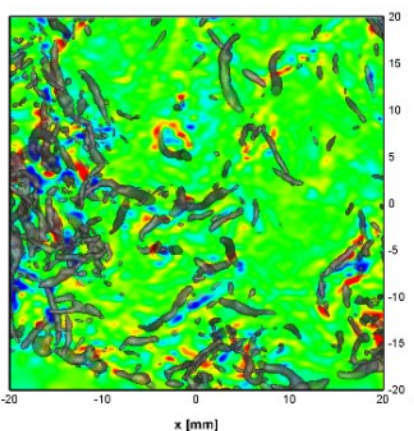

(b) $\rho_{p}=75 \%$, ANFIS

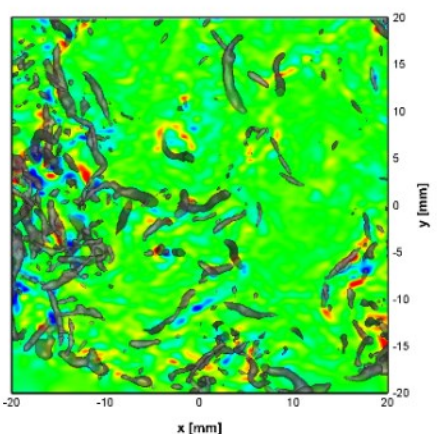

(c) $\rho_{p}=50 \%$, ANFIS

Fig. 2 (top) (a) Raw STB result (b, c) downsampled STB data as input. (bottom) Contour colored by xcomponent of acceleration and iso-surface of $Q$-criterion, $Q=5,000 \mathrm{~s}^{-2}$ from (a) FlowFit and (b, c) ANFIS. 


\section{Acknowledgements}

This research was supported by Basic Science Research Program through the National Research Foundation of Korea (NRF) funded by the Ministry of Education (2020R1A6A3A03038341) and the Korean government (MSIT) (2021R1C1C2011538). We acknowledge Daniel Schanz, Florian Huhn, Sebastian Gesemann and Andreras Schröder (German Aerospace Center) for providing the LPT and FlowFit data, Daniel Garaboa-Paz (University of Santiago de Compostella) and Eberhard Bodenschatz (Max-Planck Institute for Dynamics and Self-Organization) are acknowledged for their contributions to the experiment

\section{References}

[1] Schröder, A., et al. (2019) Measuring the full velocity gradient and dissipation rate tensor in homogeneous turbulence using Shake-The-Box and FlowFit. $17^{\text {th }}$ ETC, Torino, Italy.

[2] Gesemann, S., Huhn, F., Schanz, D., \& Schröder, A. (2016) From noisy particle tracks to velocity, acceleration and pressure fields using B-splines and penalties. In $18^{\text {th }}$ LXLASER, Lisbon, Portugal, 4-7. [3] Kim, D., Safdari, A., \& Kim, K. C. (2021) Sound pressure level spectrum analysis by combination of 4D PTV and ANFIS method around automotive side-view mirror models. Scientific Reports, 11(1), 1-15. 